\title{
Selektiver Serotoninagonist bringt den Stuhl in Gang
}

\author{
I OBSTIPATION Studien zeigen für Prucaloprid eine \\ schnelle und sichere Wirkung bei chronischer Verstopfung, \\ auch wenn diese durch Opioide verursacht ist.
}

Patienten (Prozent) mit durchschnittlich $\geq 3$ spontanen kompletten Darmentleerungen/Woche über 12 Wochen (Normalisierung)

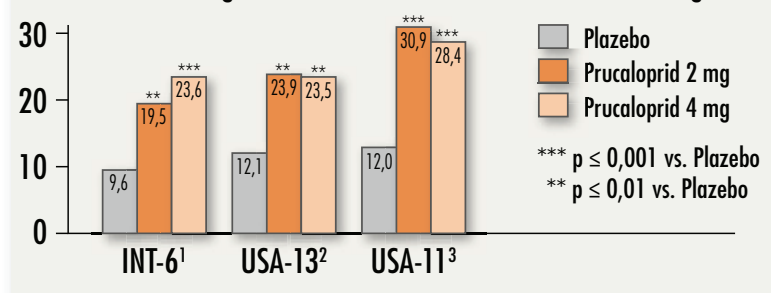

'Tack et al. Gut 2009; 58: 357 2Quigley et al. Aliment Pharmacol Ther 2009; 29: 315 ${ }^{3}$ Camilleri et al. N Engl J Med 2008; 358: 2344

Drucaloprid stimuliert hochaffin die 5HT4-Rezeptoren der Darmwand und regt die Peristaltik an", erklärte Prof. Dr. med. Stefan Müller-Lissner, Berlin. So wirkt der hochselektive Serotoninagonist (Resolor ${ }^{\circledR}$ ) direkt auf die gestörte Darmmotilität und verbessert die Darmfunktion bei Patienten mit chronischer Obstipation signifikant und schnell. Der Effekt ist in verschiedenen Studien auch bei Patienten mit opioid- oder querschnittassoziierter Obstipation nachgewiesen.

In drei Zulassungsstudien erhielten fast 2000 Patienten mit chronischer Obstipation und unzureichender Laxanzienwirkung über zwölf Wochen Prucaloprid. Unter 2 mg zeigten sich bei $24 \%$ der Patienten mindestens drei spontane komplette Darmentleerungen pro Woche, verglichen mit $11 \%$ unter Plazebo. Die

\section{Neues mobiles Biofeedback-Gerät zur Migränetherapie}

Die schwa-medico Medizinische Apparate Vertriebsgesellschaft mbH hat ein neves Biofeedbackgerät eingeführt.

VasoTrain, der mobile Coach
zur aktiven Migränetherapie,
wurde während des Schmerz-
tages im März in Frankfurt/
Main vorgestellt. Mit VasoTrain
kann der Patient unter Anlei-
tung erlernen, die Vasokonstrik-
tion willentlich zu beeinflussen
und somit die Häufigkeit von
Migräneattacken zu mindern.
schwa-medico unter www.schwa-medico.de
oder info@schwa-medico.de.
Nach Informationen der schwa-medico Apparate Vertriebs-
gesellschaft mbH

Wirkung trat innerhalb von durchschnittlich 2,5 Stunden ein, gegenüber 26 Stunden in der Plazebogruppe. Die Einnahme verbesserte auch begleitende Symptome wie Blähungen oder Unwohlsein.

Übelkeit, Diarrhö, abdominelle Schmerzen sowie Kopfschmerzen waren leichte Nebenwirkungen, die nach dem ersten Behandlungstag in der Regel wieder verschwanden. Kardiale Komplikationen sind wegen der hohen Affinität zu 5 HT4-Rezeptoren nicht zu erwarten. Bei früheren, weniger selektiven Serotonin- agonisten hatte die Wirkung auf den hERG-Kalium-Kanal kardiale Probleme ausgelöst, die dazu führten, dass die Präparate wieder vom Markt genommen werden mussten. Prucaloprid ist zunächst nur bei Frauen zugelassen, die in den Studien $90 \%$ der Probanden stellten.

Michael Koczorek

Pressekonferenz „Das Dilemma der Behandlung von chronischer Obstipation: Bietet Prucaloprid einen Ausweg?" anlässlich des 116. Kongresses der Deutschen Gesellschaft für Innere Medizin (DGIM), Wiesbaden, 12. April 2010; Veranstalter: Movetis, Belgien

\section{Anhaltende Schmerzkontrolle}

\section{CHRONISCHE SCHMERZEN Opioide können - je nach Entstehungs- mechanismus des Schmerzes - in Umkehrung des WHO-Stufen-Schemas erste Wahl sein. Gut geeignet ist buprenorphinhaltiges 7-Tage-Pflaster, das eine anhaltend stabile Analgesie bei allen Schmerzformen schafft.}

In Deutschland leiden 7,5 Millionen Menschen an chronischen Schmerzen, bei etwa einem Viertel ist der Schmerz neuropathisch. Häufig werden Betroffene gemäß WHO-Stufen-Schema mit Entzündungshemmern behandelt - trotz bekannter kardiovaskulärer und gastrointestinaler Risiken und auch wenn Entzündungsmechanismen nicht Schmerzursache sind. Erst bei intensivsten Schmerzen erfolgt die stufenweise Steigerung bis zur Opioidbehandlung. "Moderne Schmerztherapie sollte sich aber nicht an der Intensität, sondern an Entstehungs- und Chronifizierungsmechanismen orientieren", sagte Dr. Gerhard Müller-Schwefe, Göppingen. Dabei sollten Opioide wegen guter Wirksamkeit und günstigen Nebenwirkungsprofils schon früh eingesetzt werden.

Eine spezielle Rolle spielt hier Buprenorphin. Aufgrund seiner Rezeptoreigenschaften sowie der Beeinflussung spannungsabhängiger Natriumkanäle wirkt es bei nozizeptiven, neuropathischen und gemischten Schmerzen. Als 7 Tage-Pflaster (Norspan ${ }^{\circledR}$ ) appliziert sorgen gleichmäßige Wirkspiegel für anhaltende Analgesie und verbessern Parameter der Lebensqualität wie Aktivität, Stimmung oder Schlaf. Buprenorphin ist unabhängig von der Nierenfunktion einsetzbar und gut ZNS- und gastrointestinal verträglich mit niedrigem Interaktionspotenzial.

Die Dosierung muss ausreichend sein und sollte sich auch nach der Vortherapie richten, erklärte UIf Schutter aus Marl. Als Einstiegsdosis empfiehlt er $10 \mu \mathrm{g} / \mathrm{h} \mathrm{-}$ etwa nach vorheriger Behandlung mit $200 \mathrm{mg}$ Tramadol oder Tilidin. Studien zufolge brauchen über $70 \%$ der Patienten $20 \mu \mathrm{g} / \mathrm{h}$.

Michael Koczorek

Presse-Lunch Norspan ${ }^{\circledR}$ „Differenzierte Therapie mit Opioiden - was ist die Rationale?", Frankfurt/Main, 19. März 2010; Veranstalter: Grünenthal $\mathrm{GmbH}$ 